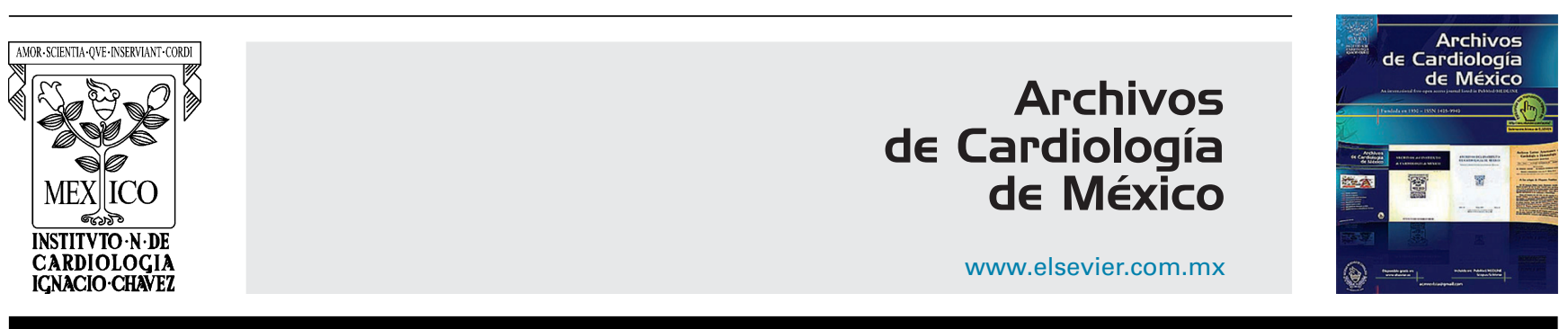

INVESTIGACIÓN CLÍNICA

\title{
Intervención coronaria percutánea del tronco no protegido de la coronaria izquierda comparada con cirugía de revascularización coronaria; experiencia de 3 años en el Instituto Nacional de Cardiología
}

\author{
Carlos López-Aguilar ${ }^{\mathrm{a}, *}$, Arturo Abundes-Velasco ${ }^{\mathrm{b}}$, Guering Eid-Lidt ${ }^{\mathrm{b}}$, \\ Yigal Piña-Reyna ${ }^{\mathrm{b}}$ y Jorge Gaspar-Hernández ${ }^{\mathrm{b}}$
}

\footnotetext{
a Servicio de Angiografía, Hospital Eugenio Espejo, Av. Gran Colombia SN y Yaguachi, Quito, Ecuador

b Departamento de Cardiología Intervencionista, Instituto Nacional de Cardiología Dr. Ignacio Chávez, Juan Badiano N1, Sección XVI, del Tlalpan, C.P. 14080, Ciudad de México, México
}

Recibido el 15 de abril de 2016; aceptado el 21 de julio de 2016

\author{
PALABRAS CLAVE \\ Intervencionismo \\ coronario \\ percutáneo; \\ Tronco no protegido \\ de coronaria \\ izquierda; \\ Cirugía de \\ revascularización \\ coronaria; \\ Seguridad; \\ Eficacia; \\ México
}

\begin{abstract}
Resumen
Antecedentes: El mejor método para el tratamiento de la revascularización de la lesión del tronco no protegido de la coronaria izquierda es un tema de creciente interés.

Método: Se realizaron un total de 2,439 intervenciones coronarias percutáneas (ICP) durante 3 años; se incluyeron los pacientes con lesión del tronco no protegido de la coronaria izquierda $(\mathrm{TCl} ; \mathrm{n}=48)$ y se compararon con pacientes a los que se realizó cirugía de revascularización coronaria (CRVC; $n=50$ ). Se analizaron los eventos cardiovasculares en fase hospitalaria y extrahospitalaria. El promedio de seguimiento fue de 16 meses.

Resultados: El riesgo cardiovascular fue mayor en pacientes con ICP; log EuroSCORE (16 \pm 21 vs. $5 \pm 6, p=0.001)$, Syntax clínico ( $77 \pm 74$ vs. $53 \pm 39, p=0.04)$. Los pacientes del grupo de ICP presentaron al ingreso con más frecuencia infarto con elevación del ST (IMCEST) y con choque cardiogénico. Los eventos hospitalarios fueron similares en ICP y CRVC (14\% vs. 18\%, $p=0.64$ ). El IMCEST fue menos frecuente en el grupo de ICP $(0 \%$ vs. $10 \%, p=0.03$. Los eventos cardiovasculares fueron menores en el grupo de ICP $(2.3 \%$ vs. $18 \%, \mathrm{p}=0.01)$ y hubo una disminución de la muerte general y cardiaca $(2.3 \%$ vs. $12 \%, p=0.08$ y $2.3 \%$ vs. $8 \%, p=0.24)$, al excluir los pacientes con choque cardiogénico como presentación. En la fase extrahospitalaria los eventos fueron similares en ICP y CRVC (15\% vs. $12 \%, p=0.46)$. La supervivencia sin eventos cardiovasculares, muerte general y cardiaca fueron comparables entre los grupos $(\log$ rank, $p=0.38, p=0.44$ y $\mathrm{p}=0.16)$.
\end{abstract}

\footnotetext{
* Autor para correspondencia. Av. Granda Centeno 034-530 y Sancho de la Carrera, CP 170104, Quito, Ecuador, Teléfono: +593 023319533. Correo electrónico: lopezaguilarc@yahoo.com.mx (C. López-Aguilar).
} 
Conclusión: Pese a que los pacientes de ICP mostraron un perfil de riesgo mayor que los de CRVC, la seguridad y eficacia intrahospitalaria y extrahospitalaria fueron similares.

(C) 2016 Instituto Nacional de Cardiología Ignacio Chávez. Publicado por Masson Doyma México S.A. Este es un artículo Open Access bajo la licencia CC BY-NC-ND (http://creativecommons. org/licenses/by-nc-nd/4.0/).

\section{KEYWORDS}

Percutaneous coronary intervention; Unprotected left main coronary artery; Coronary artery bypass grafting; Safety; Efficacy; Mexico
Percutaneous coronary intervention of unprotected left main coronary compared with coronary artery bypass grafting; 3 years of experience in the National Institute of Cardiology, Mexico

\begin{abstract}
Background: The best revascularisation method of the unprotected left main artery is a current and evolving topic.

Methods: A total of 2439 percutaneous coronary interventions $(\mathrm{PCI})$ were registered during a 3year period. The study included all the patients with $\mathrm{PCl}$ of the unprotected left main coronary $(n=48)$ and matched with patients who underwent coronary artery bypass graft (CABG) $(n=50)$. Major adverse cerebral and cardiac events (MACCE) were assessed within the hospital and in outpatients during a 16 month follow up.

Results: The cardiovascular risk was greater in the PCI group; logEuroSCORE $16 \pm 21$ vs. $5 \pm 6$, $P=.001$; clinical Syntax $77 \pm 74$ vs $53 \pm 39, P=.04$. On admission, the $P C I$ group of patients had a higher frequency of ST segment elevation myocardial infarction (STEMI) and cardiogenic shock. The MACCE were similar in both groups $(14 \%$ vs. $18 \%, P=.64)$. STEMI was less frequent in the $\mathrm{PCl}$ group $(0 \%$ vs. $10 \%, P=.03)$. Cardiovascular events were lower in the $\mathrm{PCl}$ group $(2.3 \%$ vs. $18 \%, P=.01)$, and there was a decrease in general and cardiac mortality $(2.3 \%$ vs. $12 \%, P=.08$ y $2.3 \%$ vs. $8 \%, P=.24)$, on excluding the patients with cardiogenic shock as a presentation. MACCE were similar in both groups in the out-patient phase $(15 \%$ vs. $12 \%, P=.46)$.

Survival without MACCE, general and cardiac death were comparable between groups (log rank, $P=.38, P=.44$ and $P=.16$, respectively).

Conclusion: Even though the clinical and peri-procedural risk profile of the $\mathrm{PCl}$ patients were higher, the in-hospital and out-hospital efficacy and safety were comparable with CABG.

(C) 2016 Instituto Nacional de Cardiología Ignacio Chávez. Published by Masson Doyma México S.A. This is an open access article under the CC BY-NC-ND license (http://creativecommons. org/licenses/by-nc-nd/4.0/).
\end{abstract}

\section{Introducción}

La revascularización coronaria del tronco no protegido de la coronaria izquierda $(\mathrm{TCl})$ es un área que ha evolucionado con mucha rapidez en el intervencionismo cardiaco. Durante mucho tiempo se consideró a la CRVC como el tratamiento de elección, debido al beneficio que demostró al compararla con el tratamiento farmacológico en la era previa a la llegada de los stents ${ }^{1}$. Sin embargo, en las últimas 3 décadas se ha producido un rápido desarrollo de materiales, terapia antiagregante y técnicas en la cardiología intervencionista que han mejorado la seguridad y eficacia en el tratamiento de las lesiones coronarias, previamente conocidas como no convencionales o de uso extraoficial ${ }^{2} ; y$ se ha dado paso a una continua ampliación en sus indicaciones. Este es el caso de la ICP del TCl, que ha pasado a constituirse como el método de revascularización de elección en los pacientes con enfermedad obstructiva de tronco no protegido de la coronaria izquierda, que se presentan con síndrome coronario agudo.

La ICP de TCl pasó de ser una contraindicación (tipo III) a ser una indicación lla en pacientes con angina estable y características anatómicas favorables (puntuación de Syntax $\leq 22$ o lesión cuerpo-orificio) y riesgo quirúrgico elevado (STS $\geq 5)$. Y recomendación IIb con características anatómicas y riesgo quirúrgico intermedios (Syntax 23-32 y STS $\geq 2$ ) ${ }^{3}$.

En este momento contamos con la evidencia de que el ICP del $\mathrm{TCI}$ no protegido tiene una seguridad comparable con la CRVC. El más extenso de los registros realizados hasta el momento, MAIN COMPARE ${ }^{4}$, demostró que no hay diferencia, constatado en un periodo de 5 años, en la frecuencia de muerte, infarto y accidente vascular cerebral, tanto con el uso de stents metálicos (SM) como de stents liberadores de fármaco (SLF). En cuanto a la eficacia, varios estudios demostraron de forma consistente que la ICP del TCI tiene un riesgo 5 veces mayor de revascularización de la lesión tratada, comparado con la CRVC; ya sea con $\mathrm{SM}^{4}$ como con SLF de primera ${ }^{5}$ o segunda generación ${ }^{6}$.

Los pacientes con lesión del $\mathrm{TCl}$ que padecen una enfermedad coronaria trivascular son un grupo especialmente complejo. En un subanálisis del Syntax se observó que, a pesar de tener una puntuación intermedia (23-32), estos pacientes presentan una seguridad y eficacia similar, si no mejor (menor frecuencia de enfermedad cerebro vascular) con ICP que con $\mathrm{CRVC}^{7}$, lo que ha ampliado aun más la recomendación de ICP de TCI en este grupo específico de pacientes ${ }^{8}$. Existen diferencias en la eficacia de acuerdo al tipo anatómico de la lesión del $\mathrm{TCl}$, puesto que los 
resultados de la ICP del $\mathrm{TCl}$ es significativamente mejor en los que tienen enfermedad de cuerpo-orificio comparado con el de segmento distal ${ }^{9}$.

El uso rutinario del IVUS para definir mejor la lesión de tronco, planificar la estrategia terapéutica y corroborar los resultados parece tener beneficios clínicos si se compara con la estrategia que se guía solo por la utilización de la angiografía ${ }^{10}$. En los casos de lesión de tronco que involucra la bifurcación, hay evidencia que sugiere el beneficio de utilizar la técnica de stent provisional respecto a la de dos stents $^{11}$.

Debido a estos resultados alentadores a medio y largo plazo, la ICP del $\mathrm{TCI}$ ha tenido una amplia difusión, sobre todo en los pacientes con riesgo quirúrgico elevado o que no son candidatos a CRVC. El Instituto Nacional de Cardiología es un centro de referencia nacional de pacientes con cardiopatía isquémica y cuenta con el equipo necesario, tanto quirúrgico como de intervencionismo, en el tratamiento del paciente con enfermedad de $\mathrm{TCl}$ no protegido; sin embargo, no se conocen las características clínicas, angiográficas y técnicas empleadas hasta el momento, así como la evolución a corto o medio plazo. A partir de este registro, se pretende analizar las características de la población intervenida, las técnicas empleadas así como comparar la seguridad y eficacia de la ICP respecto al CRVC en un lapso de 3 años, en fase intrahospitalaria y de seguimiento a mediano plazo.

\section{Métodos}

\section{Pacientes}

Se realizó un estudio observacional de casos y controles contemporáneos, retrospectivo y longitudinal. Se incluyeron pacientes a los que se les realizó angioplastia con 'stent' en $\mathrm{TCl}$ no protegido desde mayo del 2010 hasta mayo del 2013, registrados en la base de datos electrónica del Servicio de Hemodinámica del Instituto Nacional de Cardiología (total 2,439 intervenciones coronarias percutáneas). El grupo control lo conformaron los pacientes a quienes se les realizó CRVC durante el mismo periodo, con datos obtenidos a de los censos mensuales del departamentode cirugía cardiotorácica. De un total de 293 pacientes a los que se les realizó CRVC sin valvulopatía asociada, se seleccionaron a 50 que tuvieron lesión de $\mathrm{TCl}$. Se incluyeron los pacientes que tuvieron coronariografía diagnóstica disponible para su revisión, que estuvo a cargo de 2 cardiólogos intervencionistas expertos, adscritos al servicio de hemodinámica y de manera independiente. Se excluyeron los pacientes a los que no se les pudo realizar un seguimiento extrahospitalario, a los que padecían lesiones no ateroscleróticas del $\mathrm{TCl}$ y a los menores de 18 años. Se definió como lesión de tronco no protegido del $\mathrm{TCl}$ a la estenosis igual o mayor al 50\%; en ausencia de hemoductos venosos, puentes arteriales o de ambos. En los casos en los que realizó ultrasonido intravascular (IVUS) o reserva fraccional de flujo coronario (FFR), se consideró lesión significativa del $\mathrm{TCl}$ un área luminal mínima menor de $6 \mathrm{~mm}^{2}$ por IVUS o una FFR $\leq 0.8$. Asimismo, se definió como enfermedad trivascular la presencia de lesiones coronarias con estenosis angiográfica de $\geq 70 \%$ o $\geq$ al $50 \%$, en presencia de isquemia demostrada por algún método funcional (FFR, perfusión miocárdica o ecocardiograma con reto farmacológico) en las tres arterias coronarias principales. Se definió como bifurcación verdadera del TCI las lesiones en bifurcación, en las que la rama secundaria (circunfleja o ramo intermedio) tenían una lesión del $50 \%$ o más a menos de $5 \mathrm{~mm}$ del vaso principal (1-1-1, 0-1-1, 1-0-1 o 0-0-1 de la clasificación de Medina). Las puntuaciones de Syntax, Syntax clínico y EuroSCORE logístico se calcularon de acuerdo con lo establecido en la literatura ${ }^{11-14}$. Se definió como revascularización completa la resolución de todas las lesiones con estenosis de $\geq 50 \%$ en arterias $\geq 2 \mathrm{~mm}$ de diámetro por ICP o CRVC.

\section{Eventos}

La frecuencia de eventos se valoró tanto en la fase intrahospitalaria como extra-hospitalaria a través del seguimiento en la consulta externa y llamadas telefónicas. Se definió como evento cardiovascular mayor (MACCE, del inglés major adverse cerebral-cardiovascular event) a la combinación de eventos compuesta por: muerte por cualquier causa, muerte cardiaca, infarto cardiaco con onda $Q$, evento vascular cerebral y revascularización del vaso tratado. Se definió la muerte cardiaca como la secundaria al síndrome coronario agudo, insuficiencia o arritmia cardiaca; evento cerebrovascular como un déficit neurológico transitorio o que persiste durante $>24$ horas, que sea registrado en el expediente clínico o por interrogatorio dirigido durante el seguimiento; revascularización del vaso tratado como nueva ICP o CRVC en la arteria coronaria tratada; infarto con onda $\mathrm{Q}$ como dolor torácico característico con presencia de ondas $\mathrm{Q}$ patológicas en 2 derivaciones continuas del ECG; infarto no $Q$ como elevación de 5 veces los niveles de troponina I o creatincinasa $M B(C K-M B)$ respecto al rango de normalidad reportado por el laboratorio en caso de la ICP, o 10 veces mayor en caso de CRVC. En caso de estar previamente elevados, se consideró significativa un aumento del $20 \%$ respecto a una determinación previa. Para definir trombosis del stent definitiva o definitiva-probable se utilizó la clasificación de la ARC (Academic Research Consortium).

\section{Análisis estadístico}

Las variables cuantitativas se expresaron como promedios \pm -desviaciones estándar o media y rangos intercuartílicos, según la distribución evaluada por la prueba de KolmogorovSmirnov. Para determinar las diferencias entre variables continuas se utilizó la prueba de ANOVA. Las variables cualitativas se expresaron con porcentajes y las diferencias se evaluaron con la prueba de la Ji al cuadrado o prueba de Fisher, según corresponda. Se valoró la supervivencia sin MACCE con curvas de Kaplan Meier y su significancia estadística con la prueba de rango logarítmico. Los predictores de MACCE se evaluaron con regresión logística lineal, uni y multivariable. Se consideró estadísticamente significativo un valor de $p<0.05$.

\section{Resultados}

En el periodo transcurrido desde mayo del 2010 hasta mayo del 2013 se realizaron 2,439 intervenciones coronaria percutáneas (ICP), de las cuales 73 (3\%) fueron por lesión del 
Tabla 1 Características basales

\begin{tabular}{|c|c|c|c|c|}
\hline Variable & $\begin{array}{l}\text { Total }(\%) \\
\mathrm{n}=98\end{array}$ & $\begin{array}{l}\text { ICP (\%) } \\
n=48\end{array}$ & $\begin{array}{l}\text { CRVC (\%) } \\
n=50\end{array}$ & $\mathrm{p}$ \\
\hline Hombres & 78 (79) & $35(73)$ & $43(86)$ & 0.10 \\
\hline Edad & $66 \pm 11$ & $65 \pm 12$ & $66 \pm 10$ & 0.67 \\
\hline \multicolumn{5}{|l|}{ Factores de riesgo } \\
\hline Hipertensión arterial & $55(56)$ & $25(52)$ & $30(60)$ & 0.27 \\
\hline Diabetes mellitus & $41(42)$ & $23(48)$ & $18(36)$ & 0.16 \\
\hline Tabaquismo & $26(26)$ & $10(20)$ & $16(32)$ & 0.15 \\
\hline Dislipidemia & $44(45)$ & $22(46)$ & $22(44)$ & 0.50 \\
\hline Creatinina & $1.2 \pm 0.8$ & $1.3 \pm 1.1$ & $0.9 \pm 0.2$ & 0.05 \\
\hline Depuración $\mathrm{Cr}, \mathrm{ml} / \mathrm{min}$ & $73 \pm 29$ & $70 \pm 34$ & $77 \pm 24$ & 0.29 \\
\hline Depuración $\mathrm{Cr}<60$ & $31(31)$ & $19(39)$ & $12(24)$ & 0.09 \\
\hline EAC previa & $46(47)$ & $22(46)$ & $24(48)$ & 0.49 \\
\hline IM & $40(40)$ & $18(37)$ & $22(44)$ & 0.51 \\
\hline ICP & $15(15)$ & $11(23)$ & $4(8)$ & 0.03 \\
\hline Presentación clínica & & & & 0.03 \\
\hline Angina estable & $46(47)$ & $17(35)$ & $29(58)$ & \\
\hline SICA $\sin S T$ & $32(33)$ & $16(33)$ & $16(32)$ & \\
\hline SICA con ST & $14(14)$ & 9 (19) & $5(10)$ & \\
\hline Choque cardiogénico & $6(6)$ & $6(12)$ & 0 & \\
\hline FEVI \% & $47 \pm 14$ & $44 \pm 14$ & $51 \pm 13$ & 0.01 \\
\hline$F E V I<40 \%$ & $29(30)$ & $20(42)$ & $9(18)$ & $<0.01$ \\
\hline EurOSCORE log & $10 \pm 16$ & $16 \pm 21$ & $5 \pm 6$ & 0.001 \\
\hline Syntax & $30 \pm 11$ & $28 \pm 12$ & $32 \pm 10$ & 0.11 \\
\hline Syntax $\geq 33$ & $38(38)$ & $16(33)$ & $22(44)$ & 0.27 \\
\hline Syntax Clínico & $65 \pm 60$ & $77 \pm 74$ & $53 \pm 39$ & 0.04 \\
\hline
\end{tabular}

EAC: enfermedad arterial coronaria; IM: infarto de miocardio; SICA: síndrome isquémico coronario agudo; FEVI: fracción de expulsión del ventrículo izquierdo.

En negrita, las variables que demostraron diferencia estadísticamente significativa.

$\mathrm{TCl}$. Se excluyeron 18 casos de $\mathrm{TCl}$ protegido, 4 con lesiones no ateroscleróticas ( 3 disecciones y 1 caso de compresión extrínseca del $\mathrm{TCl}$ ) y 4 pacientes con lesión aterosclerótica del $\mathrm{TCl}$, en los que no se completó el seguimiento; por lo que, para el análisis, se incluyeron 48 pacientes en el grupo de ICP. En el mismo periodo se realizaron 293 CRVC, de las que $67(2.2 \%)$ correspondieron a CRVC por lesión del TCI. De las 67 CRVC, 15 no tuvieron coronariografía en el archivo del Instituto y en 2 no se completó el seguimiento extrahospitalario, por lo que se incluyeron un total de 50 pacientes en el análisis. La pérdida en el seguimiento en los dos grupos fue de 6 pacientes (5.7\%).

El promedio de edad fue de 66 años, con un predominio de varones en los dos grupos. El diagnóstico de presentación más frecuente en el grupo de ICP fue el de infarto de miocardio con elevación del segmento ST y choque cardiogénico. El Syntax clínico y el Euro-score fueron mayores en los pacientes con ICP y la función ventricular estuvo más deteriorada comparados con los paciente con CRVC. La puntuación de Syntax fue similar en los dos grupos ( $28 \pm 12$ vs. $32 \pm 10$ puntos, $p=0.11$ ). El grupo de ICP mostró una tendencia a una menor depuración de creatinina que el grupo de CRVC. (tabla 1). La duración del seguimiento fue significativamente mayor en la CRVC ya que, como se observa en la figura 1, la cantidad de CRVC fue mayor en el 2010 que en años posteriores, mostrando una relación inversa a la ICP, que se incrementó de 2 casos en el 2010 a 27 en el 2012.
La prevalencia de la enfermedad trivascular y de la lesión distal del $\mathrm{TCl}$ fue numéricamente mayor en el grupo de CRVC. La presencia de trombo fue exclusiva del grupo de ICP debido a la mayor frecuencia de síndrome coronario agudo como presentación clínica. (tabla 2). En el $12 \%$ del grupo de ICP se usó IVUS y solo en un caso balón de corte. En las lesiones de orificio-cuerpo del $\mathrm{TCl}$ la técnica de 1 stent fue la mas utilizada (95\%). En las lesiones distales del $\mathrm{TCl}$ la técnica de stent provisional y la de 2 stents tuvieron la misma frecuencia (37\%). En el grupo con lesiones de bifurcación verdadera predominó la de 2 stents (50\%), donde

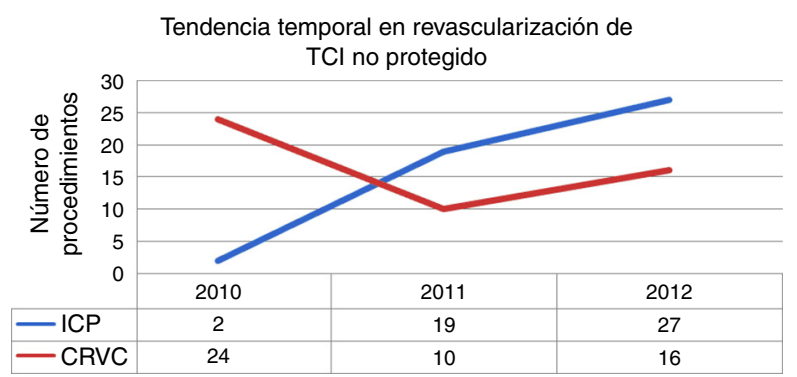

Figura 1 Tendencia temporal en el método de revascularización de $\mathrm{TCl}$ no protegido entre mayo de 2010 y mayo de 2012. CRVC: cirugía de revascularización coronaria, ICP: intervención coronaria percutánea. 
Tabla 2 Variables periprocedimiento

\begin{tabular}{|c|c|c|c|c|}
\hline Variable & $\begin{array}{l}\text { Total (\%) } \\
\mathrm{n}=98\end{array}$ & $\begin{array}{l}\text { ICP (\%) } \\
n=48\end{array}$ & $\begin{array}{l}\text { CRVC (\%) } \\
n=50\end{array}$ & $\mathrm{p}$ \\
\hline Enf. Trivascular & $62(63)$ & $27(56)$ & $35(70)$ & 0.15 \\
\hline DA ostial & $50(51)$ & $24(50)$ & $26(52)$ & 0.84 \\
\hline Tronco distal & $63(64)$ & $27(56)$ & $36(72)$ & 0.10 \\
\hline Bifurcación verdadera & $39(40)$ & $18(37)$ & $21(42)$ & 0.64 \\
\hline Trombo & $8(8)$ & $8(17)$ & 0 & $<0.01$ \\
\hline Calcificación grave & $9(9)$ & $6(12)$ & $3(6)$ & 0.22 \\
\hline Balón de corte & & $1(2)$ & & \\
\hline IVUS & & $6(12)$ & & \\
\hline Endoférula directa & & $7(15)$ & & \\
\hline \multicolumn{5}{|l|}{ Técnica } \\
\hline En TCl ostial-cuerpo & & n 21 & & \\
\hline 1 stent & & $20(95)$ & & \\
\hline 2 stents & & $1(5)$ & & \\
\hline En TCl distal & & n 27 & & \\
\hline Stent provisional & & $10(37)$ & & \\
\hline 1 stent & & $7(25)$ & & \\
\hline 2 stents & & $10(37)$ & & \\
\hline En Bif verdadera & & n 18 & & \\
\hline Stent provisional & & $4(22)$ & & \\
\hline 2 stents & & $9(50)$ & & \\
\hline Técnica 2 stents & & n 11 & & \\
\hline TAP & & $4(37)$ & & \\
\hline T stenting & & $3(27)$ & & \\
\hline Culotte & & $2(18)$ & & \\
\hline SKS & & $1(9)$ & & \\
\hline Mini-Crush & & $1(9)$ & & \\
\hline SLF & & $28(58)$ & & \\
\hline SLF $2^{\mathrm{a}}$ generación & & $15(31)$ & & \\
\hline SM & & $15(31)$ & & \\
\hline Kissing final & & $11(23)$ & & \\
\hline \multirow[t]{2}{*}{ Diámetro stent } & & 3.6 & & \\
\hline & & $\pm 0.92 \mathrm{~mm}$ & & \\
\hline Longitud stent & & $20 \pm 8 \mathrm{~mm}$ & & \\
\hline Presión ATM & & $15 \pm 4$ & & \\
\hline Inhibidor IIb IIla & & $8(16)$ & & \\
\hline BIAC & $26(26)$ & $14(29)$ & $12(24)$ & 0.56 \\
\hline Vasos tratados & $2.16 \pm 0.9$ & $1.98 \pm 0.8$ & $2.34 \pm 0.9$ & 0.04 \\
\hline Revas completa & $41(41)$ & $19(40)$ & $21(52)$ & 0.80 \\
\hline Lesiones tratadas & $2.2 \pm 0.9$ & $2.1 \pm 0.9$ & $2.4 \pm 0.9$ & 0.11 \\
\hline Puentes venosos & & & $1.4 \pm 0.9$ & \\
\hline Puentes arteriales & & & $1.0 \pm 0.7$ & \\
\hline AMII a DA & & & $38(76)$ & \\
\hline CEC & & & $38(76)$ & \\
\hline
\end{tabular}

AMII: puente de arteria mamaria interna izquierda; BIAC: balón intra aórtico de contrapulsación; CEC: circulación extracorpórea; TAP: T and protrusión; SKS: simultaneous kissing stent.

En negrita, las variables que demostraron diferencia estadísticamente significativa.

las técnicas en bifurcación de T stenting, T-and-protrusion (TAP) y Culotte fueron las más frecuentes con el 37, 27 y $18 \%$, respectivamente. En la mayoría de las ICP se utilizaron SLF (58\%), el $31 \%$ de los cuales fue de segunda generación. La cantidad de vasos tratados fue significativamente mayor en el grupo de CRVC (2.3 vs. $1.9, p=0.04)$. Sin embargo, el porcentaje de revascularización completa fue comparable (52 vs. $40 \%, p=0.80$ ). Se implantó de promedio 1 puente arterial por cada paciente y el $76 \%$ recibió un injerto de arteria mamaria interna. El uso de balón de contrapulsación aórtico (BIAC) periprocedimiento fue muy similar en los dos grupos ( 29 vs. $24 \%, p=0.56)$.

\section{Eventos intrahospitalarios}

El infarto periprocedimiento por criterios enzimáticos fue más frecuente en el grupo de CRVC (48\% vs. $4 \%, p=<0.01)$. 
Tabla 3 Eventos

\begin{tabular}{|c|c|c|c|c|}
\hline Variable & $\begin{array}{l}\text { Total (\%) } \\
\mathrm{n}: 98\end{array}$ & ICP (\%) n: 48 & $\begin{array}{l}\text { CRVC (\%) n: } \\
50\end{array}$ & $\mathrm{p}$ \\
\hline \multicolumn{5}{|l|}{ Hospitalarios } \\
\hline MACCE & $16(16)$ & $7(14)$ & $9(18)$ & 0.64 \\
\hline Muerte general & $13(13)$ & $7(14)$ & $6(12)$ & 0.70 \\
\hline Muerte cardiaca & $10(10)$ & $6(12)$ & $4(8)$ & 0.34 \\
\hline IM no Q & $26(26)$ & $2(4)$ & $24(48)$ & $<0.01$ \\
\hline IM Q & $5(5)$ & 0 & $5(10 \%)$ & 0.03 \\
\hline RVT & $2(2)$ & 0 & $2(4)$ & 0.25 \\
\hline EVC & 0 & 0 & 0 & - \\
\hline Reoperación por sangrado & $5(5)$ & 0 & $5(10 \%)$ & - \\
\hline Estancia hosp (dias) & $20 \pm 15$ & $9 \pm 7$ & $30 \pm 13$ & $<0.001$ \\
\hline \multicolumn{5}{|l|}{ Extrahospitalarios } \\
\hline MACCE & $12(13)$ & $7(15)$ & $6(12)$ & 0.46 \\
\hline Muerte general & 7 (7) & $4(8)$ & $3(6)$ & 0.47 \\
\hline Muerte cardiaca & $4(4)$ & $3(6)$ & $1(2)$ & 0.29 \\
\hline IM Q & $4(4)$ & $3(6)$ & $1(2)$ & 0.27 \\
\hline RVT & $6(6)$ & $3(7)$ & $3(6)$ & 0.60 \\
\hline EVC & $2(2)$ & $1(2)$ & $1(2)$ & 0.74 \\
\hline Trombosis del stent & - & $4(8)$ & - & - \\
\hline Trombosis definitiva & - & $1(2)$ & - & - \\
\hline Trombosis probable & - & $3(6)$ & - & - \\
\hline Temprana < 30dias & - & $3(6)$ & - & - \\
\hline Seguimiento (meses) & $16 \pm 12$ & $11 \pm 9$ & $20 \pm 13$ & $<0.001$ \\
\hline
\end{tabular}

RVT: revascularización del vaso tratado; EVC: evento vascular cerebral; IM: infarto de miocardio.

En negrita, las variables que demostraron diferencia estadísticamente significativa.

Por este motivo, se realizó también un subanálisis en el que no se consideró el infarto sin onda $\mathrm{Q}$ como parte del compuesto de eventos cardiovasculares mayores (MACCE). La frecuencia de MACCE fue similar en los dos grupos (tabla 3). De manera independiente, la muerte por cualquier causa $(14 \%$ vs. $12 \% \mathrm{p}=0.07)$; muerte por causa cardiaca $(12 \%$ vs. $8 \%, p=0.38)$ y revascularización del vaso tratado ( 0 vs. $4 \%$, $p=0.25$ ) fueron similares en los dos grupos. De los 7 pacientes que fallecieron en el grupo de ICP, 4 se encontraban en choque cardiogénico al momento del ingreso y mostraron deterioro progresivo, 1 falleció por choque séptico y 2 fallecieron por trombosis del "stent"' (una trombosis definitiva y una probable). Por lo tanto, la progresión del choque cardiogénico fue la causa más frecuente de muerte en el grupo de ICP (4 de 7 pacientes, 57\%). Se utilizó inhibidor de la glucoproteína IIb, Illa en el 16\% del grupo de ICP y el inhibidor del receptor $\mathrm{P} 2 \mathrm{Y} 12$ en todos fue el Clopidogrel. No se realizaron estudios de actividad plaquetaria residual en los pacientes del registro.

La ICP es el tratamiento de elección en los pacientes con choque cardiogénico y este diagnóstico es un factor de riesgo asociado a muy mal pronóstico intrahospitalario, por lo que se realizó un subanálisis sin los pacientes que se presentaron con choque cardiogénico. Se encontró que la frecuencia de MACCE, muerte general y muerte cardiaca fueron de $2.3 \%$ vs. $18 \%(p=0.01), 2.3 \%$ vs. $12 \%(p=0.08)$ y $2.3 \%$ vs. $8 \%(p=0.24)$ para ICP y CRVC, respectivamente. La tasa de revascularización del vaso tratado fue del $4 \%$ para la CRVC, que correspondió a dos pacientes con infarto periprocedimiento con elevación del segmento ST que condicionó inestabilidad hemodinámica y requirieron ICP de rescate. En los dos casos, se encontró oclusión aguda de los puentes coronarios. En el grupo de ICP no se observó infarto con elevación del ST. En el grupo de CRVC se encontraron 5 eventos de sangrado mayor que requirieron reoperación urgente (10\%). La estancia hospitalaria fue significativamente mayor en el grupo de CRVC (30 vs. 9 días $p=<0.001$ ).

\section{Eventos extrahospitalarios}

La frecuencia de MACCE, muerte por cualquier causa, muerte cardiaca revascularización del vaso tratado fue comparable entre los dos grupos (figs. 2-5). El infarto con onda $Q$ fue más frecuente en el grupo de ICP (6 vs. $2 \%$ ), sin alcanzar una significancia estadística. La frecuencia de trombosis definitiva del stent'fue del $2 \%$, la misma que aconteció en un paciente con infarto de miocardio con elevación del segmento ST 25 días después de la ICP. Se corroboró el diagnóstico por coronariografía y se realizó intervencionismo de urgencia con resultado exitoso. Se observaron 3 casos de trombosis probable del stent.;el primer paciente presentó choque cardiogénico y fibrilación ventricular refractaria a reanimación, no presentó cambios electrocardiográficos previos ni se realizó coronariografía; tanto el segundo como el tercer pacientes presentaron dolor torácico típico en los primeros 30 días después de egresados del hospitaly fallecieron antes de su llegada al hospital. Todos los eventos de trombosis definitiva y probable fueron tempranos (<30 días). 


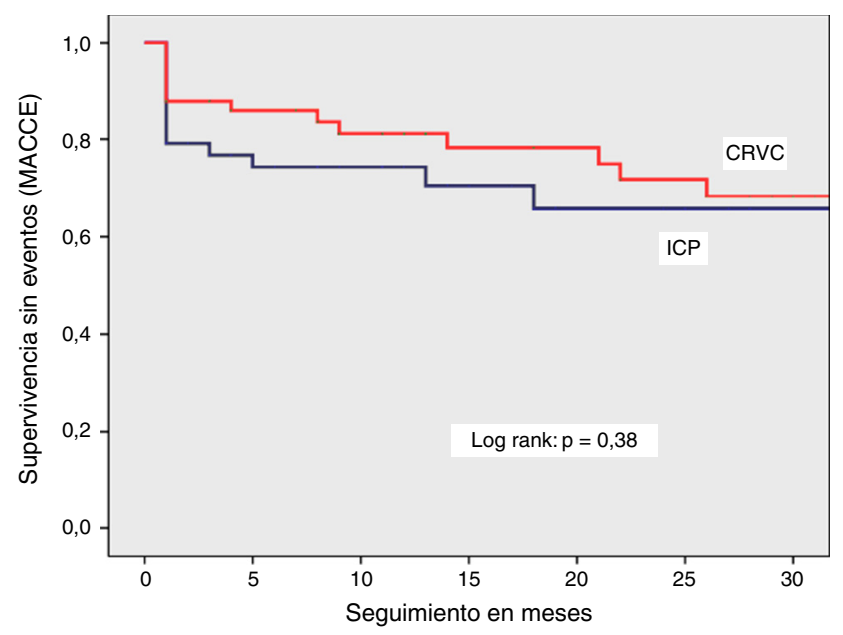

Figura 2 Supervivencia sin eventos (MACCE). CRVC: cirugía de revascularización coronaria, MACCE: eventos cardiovasculares mayores, ICP: intervención coronaria percutánea.

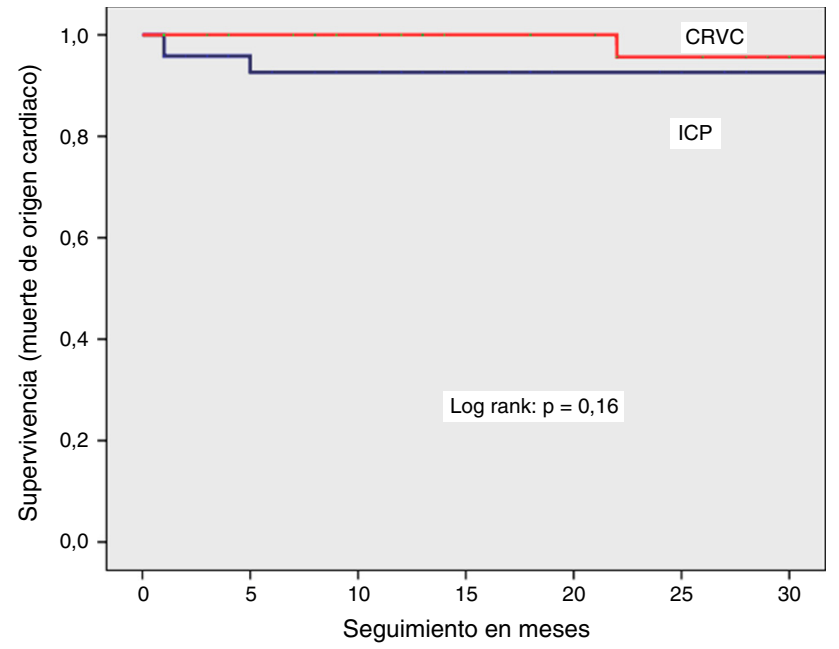

Figura 3 Supervivencia sin muerte de origen cardiaco. CRVC: cirugía de revascularización coronaria, ICP: intervención coronaria percutánea.

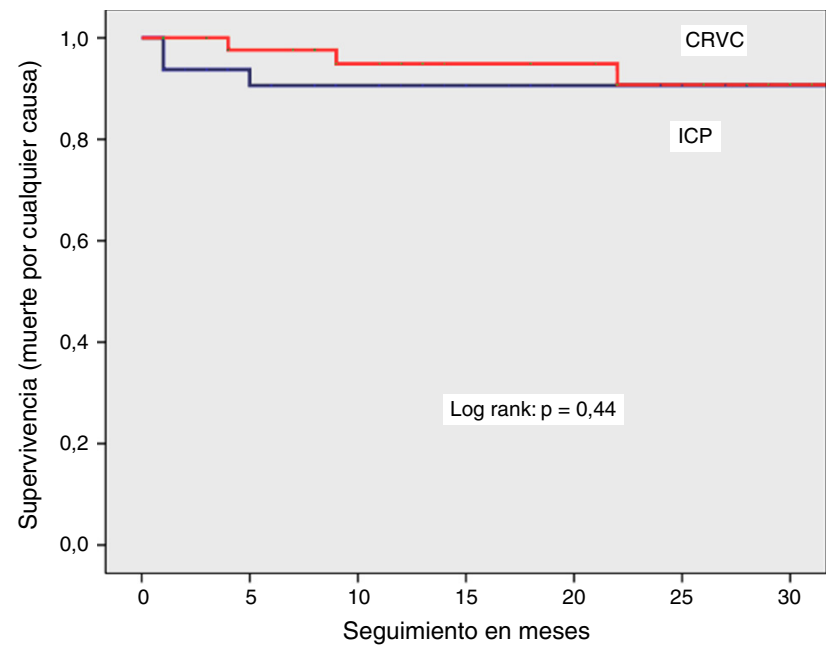

Figura 4 Supervivencia sin muerte por cualquier causa. CRVC: cirugía de revascularización coronaria, ICP: intervención coronaria percutánea.

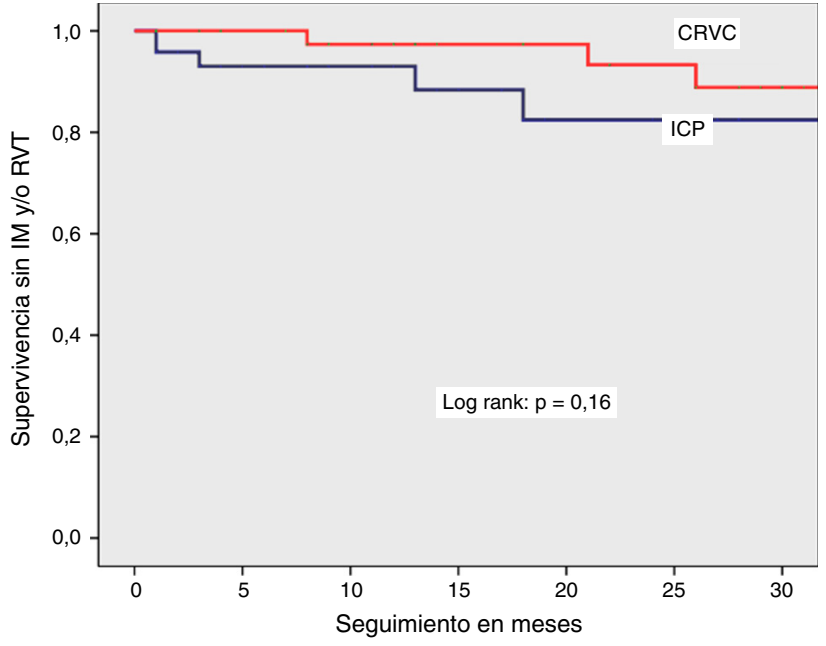

Figura 5 Supervivencia sin infarto de miocardio o revascularización del vaso tratado. CRVC: cirugía de revascularización coronaria, ICP: intervención coronaria percutánea, IM: infarto de miocardio; RVT: revascularización del vaso tratado.

Se observó una sobrevida similar sin MACCE (85\% vs. $88 \%$, log rank $p=0.38)$, muerte por cualquier causa $(92 \%$ vs. $94 \%$, log rank $p=0.44$ ) o por causas cardiacas $(94 \%$ vs. $98 \%$, log rank $\mathrm{p}=0.16$ ) en el grupo de ICP y CRVC, respectivamente. La combinación de eventos cardiovasculares (MACCE) se presentó con más frecuencia en el primer mes de evolución y tendieron a estabilizarse en los meses posteriores para los dos grupos. La sobrevida libre de infarto con onda $Q$ y revascularización del vaso tratado mostró una tendencia a ser menor en el grupo de ICP, sin significancia estadística.

En la regresión logística univariable las variables que se asociaron con MACCE fueron: dislipidemia, tipo de presentación clínica, EuroSCORE logístico, syntax clínico, número de puentes arteriales, revascularización completa y el uso de SLF en la ICP. En el análisis multivaraible la única variable que se asoció con mayor frecuencia de MACCE fue la puntuación de EuroSCORE logístico (RR 1.072, IC 95\%, 1.013-1.13).

\section{Discusión}

Presentamos un registro de un centro de referencia nacional en el que se comparan las características clínicas, angiográficas y técnicas, así como la seguridad y eficacia del intervencionismo percutáneo con la CRVC del $\mathrm{TCl}$ en un periodo de 3 años. Se incluyeron a todos los pacientes intervenidos por lesiones ateroscleróticas y en varios escenarios clínicos, incluido el síndrome coronario agudo y choque cardiogénico, por lo que es un reflejo de la revascularización del $\mathrm{TCl}$ no protegido en la práctica habitual de este centro.

Las características anatómicas fueron similares en los dos grupos tomando como referencia el puntaje de Syntax y la prevalencia de enfermedad trivascular. Sin embargo, como era de esperar, la frecuencia de lesión distal del tronco fue más frecuente en el grupo de CRVC sin alcanzar significancia estadística, probablemente por la poca cantidad de pacientes incluidos. Por otro lado, se demostró que las condiciones clínicas de los pacientes a los que se realizan ICP fueron significativamente más críticas, con función ventricular más 
deteriorada, depuración de creatinina más baja y riesgo quirúrgico más elevado. Esto está estrechamente relacionado con el diagnóstico al ingreso; el infarto con elevación del ST y el choque cardiogénico predominantes en el grupo de ICP. Esto nos parece concordante con las recomendaciones para la revascularización en $\mathrm{TCl}$ en infarto agudo de miocardio (recomendación clase I) ${ }^{3}$.

En cuanto a las variables del procedimiento, llama la atención la baja frecuencia con la que se utilizó IVUS para guiar la ICP, a pesar de que hay evidencias que sugieren su beneficio clínico ${ }^{10}$. Creemos que la baja utilización de IVUS en este registro es debido al incremento significativo de los costos que implica su utilización en una institución pública. Era de prever que el uso de un stent sea más frecuente en las lesiones del orificio y cuerpo, mientras que las técnicas de stent provisional y 2 stents sean más frecuentes en lesiones distales del $\mathrm{TCl}$. El porcentaje de técnica "compleja" o de 2 stents es similar a la de otros registros $(35 \%)^{11,15}$.

En el grupo de CRVC se observó una mayor cantidad de vasos tratados por paciente, pero esto no se reflejó en una mayor tasa de revascularización completa, que es comparable con las del grupo de ICP. Este hallazgo es muy importante pues uno de los criterios más importantes y una de las ventajas potenciales de la CRVC es una mayor frecuencia de revascularización completa ${ }^{4-6}$.

En nuestro registro encontramos que la frecuencia de muerte, infarto con onda $\mathrm{Q}$, revascularización del vaso tratado y accidente cerebrovascular fue similar con ICP y CRVC, tanto intrahospitalario como a mediano plazo, lo cual concuerda con registros internacionales (MAIN COMPARE y Registro DELTA) ${ }^{4,16}$, estudios clínicos aleatorizados (Precombat y Precombat 2$)^{5,6}$ y los metaanálisis ${ }^{17}$ más representativos en el tema.

La frecuencia de infarto de miocardio por criterios enzimáticos que se encuentra en los pacientes de CRVC en el ámbito intrahospitalario supera con mucho a los pacientes de ICP. El incluirlos en el compuesto de MACCE favorecería de forma significativa los resultados de ICP, aún sin tener la certeza de que esto se vea reflejado en un beneficio clínicamente relevante. Si bien el infarto periprocedimiento ha demostrado tener efecto pronóstico, esto se limita a comparaciones entre pacientes que se revascularizan por el mismo método, concretamente en el ámbito de ICP ${ }^{18}$. Sin embargo, en este registro se comparan dos métodos de revascularización entre sí. La cirugía es invasiva y causa lesión miocárdica no isquémica, inherente al trauma quirúrgico, y cardioplejía, lo que se ve reflejado en valores más elevados de biomarcadores en el postoperatorio inmediato; cuya importancia pronóstica no está aún aclarada.

En la literatura, la ventaja de la CRVC sobre la ICP radica en la consistente menor necesidad de revascularización del vaso tratado, para lo cual nuestro registro muestra una tendencia.

Si comparamos la frecuencia de eventos con el MAIN COMPARE, observamos en nuestro estudio una mayor frecuencia de muerte por cualquier causa (14\%), muerte cardiaca $(9 \%)$, infarto de miocardio (1\%) y EVC $(1.9 \%)^{4}$; que podría explicarse por el perfil de riesgo más elevado en nuestro estudio, puesto que, en el MAIN COMPARE, se excluyeron los pacientes con choque cardiogénico e infarto con elevación del ST.
En el MAIN COMPARE el promedio de edad fue menor (61 años); la función ventricular más conservada, la frecuencia de diabetes mellitus (32\%), falla renal crónica (3\%) y enfermedad trivascular (24\% en ICP y $57 \%$ en CRVC) fue menor que en nuestra población. Sin embargo, la tasa de revascularización del vaso tratado (9\%) y la trombosis definitiva del stent $(1.5 \%)$ fueron similares.

El registro multicéntrico Delta es el más parecido a nuestro estudio, debido a que incluyó pacientes con infarto con elevación del ST y analizó aspectos técnicos de la CRVC e $I C P^{16}$. En el resgistro Delta el perfil de riesgo fue también menor, tuvo menos diabéticos $(30 \%)$, pacientes con insuficiencia renal (6\%), insuficiencia cardiaca previa (3\%) y enfermedad trivascular (24\% y $57 \%$ en grupo de ICP y CRVC respectivamente). El EuroSCORE en el grupo de ICP fue menor a nuestro registro (16 vs. 5 puntos), mientras que en los de CRVC fue igual (5 puntos). La complejidad anatómica en el registro Delta fue similar a la nuestra, con compromiso del tronco distal del $60 \%$ al $64 \%$, de los cuales un $40 \%$ correspondió a bifurcación verdadera. A diferencia de nuestro estudio, el puntaje Syntax fue mayor en el grupo de CRVC (28 vs. 38 respectivamente). En definitiva, en nuestro registro los pacientes de ICP fueron clínica y anatómicamente más complejos que los reportados en la literatura, mientras que los de CRVC son similares.

En referencia a los aspectos técnicos, en el Delta se utilizó en todos los casos SLF y con más frecuencia IVUS (33\%), "Kissing Balloon" final (44\%) y técnica de 2 stents (45\%). El uso de BIAC fue menor en el Delta (7\%).

En el grupo de CRVC se encuentran las diferencias más significativas entre los dos estudios. La revascularización completa fue del $98 \%$ mientras que en nuestro estudio fue del $52 \%$. Sin embargo, hay que considerar que en nuestro registro se utilizó el criterio clásico y puramente anatómico para su definición, es decir, lesiones con estenosis del $\geq 50 \%$ que en ocasiones no tienen repercusión hemodinámica. $\mathrm{El}$ promedio de vasos tratados por pacientes fue de 3.7, comparado con el 2.4 en nuestro estudio. La CRVC sin bomba de circulación extracorpórea fue del 13\%, mientras que en nuestro caso fue mayor (24\%), lo que explicaría, en parte, la menor frecuencia de puentes realizados y la tasa de revascularización completa en el grupo quirúrgico. La estancia hospitalaria en el grupo de CRVC fue más corta en el Delta (12 vs. 20 días).

Los eventos hospitalarios fueron mayores en nuestro estudio, mientras que en el seguimiento extrahospitalario fueron menos frecuentes comparados con el registro Delta, lo que podría explicarse por la mayor comorbilidad y riesgo cardiovascular en nuestro estudio (tabla 4). Cuando se excluyeron los pacientes en choque cardiogénico, la frecuencia de MACCE hospitalarios, muerte general y muerte cardiaca fueron muy similares para el grupo de ICP. En el grupo de CRVC, los eventos fueron más frecuentes que en el Delta. La trombosis definitiva del stent fue similar a lo reportado en la literatura ${ }^{4-6,16}$. Sin embargo, la trombosis definitiva-probable fue mayor a la esperada, con una presentación exclusivamente temprana (<30 días), lo que podría estar relacionado con el estado más crítico de los pacientes, con síndrome coronario agudo como presentación en el grupo de ICP. Además creemos que aspectos técnicos de la intervención como una infrautilización de IVUS o el no 
Tabla 4 Comparación de eventos intra y extrahospitalarios con el Registro Delta ${ }^{16}$

\begin{tabular}{|c|c|c|c|c|}
\hline \multirow[t]{2}{*}{ Variable } & \multicolumn{2}{|c|}{ Población estudiada } & \multicolumn{2}{|c|}{ Registro Delta } \\
\hline & ICP (\%) & CRVC (\%) & ICP (\%) & CRVC (\%) \\
\hline \multicolumn{5}{|l|}{ Hospitalarios } \\
\hline Muerte general & 14 & 12 & 2.2 & 3.2 \\
\hline Muerte cardiaca & 12 & 8 & 1.7 & 2.2 \\
\hline Infarto de miocardio & 4 & 48 & 4 & 24 \\
\hline RVT & 0 & 4 & 0.8 & 0.3 \\
\hline EVC & 0 & 0 & 0.2 & 1.3 \\
\hline \multicolumn{5}{|l|}{ Extrahospitalarios } \\
\hline MACCE & 15 & 12 & 35 & 23.5 \\
\hline Muerte general & 8 & 6 & 14 & 11.4 \\
\hline Muerte cardiaca & 6 & 2 & 7.5 & 6.8 \\
\hline$I M Q$ & 6 & 2 & 3.7 & 4 \\
\hline RVT & 7 & 6 & 15.5 & 5.2 \\
\hline EVC & 2 & 2 & 1.6 & 2.9 \\
\hline \multicolumn{5}{|l|}{ Trombosis del stent } \\
\hline Trombosis definitiva & 2 & - & 1 & - \\
\hline Trombosis probable & 6 & - & 1.6 & - \\
\hline Temprana $<30$ dias & 6 & - & 0.48 & - \\
\hline Seguimiento (meses) & 16.2 & & 42.2 & \\
\hline
\end{tabular}

usar antiagregantes más potentes como Prasugrel o Ticagrelor, pudo haber influido en alto porcentaje de trombosis probable.

El perfil de seguridad y eficacia a mediano plazo se muestra muy favorable al compararlo con el Delta; sin embargo, hay que tener presente que es una muestra pequeña con un seguimiento casi tres veces menor.

\section{Limitaciones del estudio}

Este estudio tiene las limitaciones propias de los registros, donde la comparación entre grupos no tiene la aleatorización propia de estudios clínicos prospectivos. De igual manera, los resultados aquí encontrados reflejan la experiencia y desempeño de un solo centro, sin poder extrapolarse a otras instituciones o regiones del país.

\section{Conclusiones}

La ICP de TCI no protegido corresponde a un $2 \%$ de las intervenciones coronarias en el Instituto Nacional de Cardiología, con una tendencia rápida a incrementar su frecuencia en corto tiempo (3 años). Las características clínicas de los pacientes de ICP son más críticas que en el caso de CRVC, con una complejidad anatómica comparable. Los aspectos técnicos de la intervención son contemporáneos en relación con la literatura, con la excepción de la infrautilización del IVUS en nuestra población. La seguridad intrahospitalaria y, a mediano plazo de la ICP del TCI, es similar a la de la CRVC, e incluso parece ser mejor que la CRVC si se excluyen a los pacientes en choque cardiogénico al ingreso. La eficacia a corto y mediano plazo es similar a la CRVC y respecto a lo publicado en la literatura.

\section{Responsabilidades éticas}

Protección de personas y animales. Los autores declaran que para esta investigación no se han realizado experimentos en seres humanos ni en animales.

Confidencialidad de los datos. Los autores declaran que en este artículo no aparecen datos de pacientes.

Derecho a la privacidad y consentimiento informado. Los autores declaran que en este artículo no aparecen datos de pacientes.

\section{Declaración de conflictos de interés y financiamiento}

Los autores de este estudio declaramos que no tenemos conflictos de interés ni financiamiento alguno para este estudio.

\section{Bibliografía}

1. Chaitman BR, Fisher LD, Bourassa MG, et al. Effect of coronary bypass surgery on survival patterns in subsets of patients with left main coronary artery disease. Report of the Collaborative Study in Coronary Artery Surgery (CASS). Am J Cardiol. 1981;48:765-77.

2. Tu JV, Bowen J, Chiu M, et al. Effectiveness and safety of drugeluting stents in Ontario. N Engl J Med. 2007;357:1393-402.

3. Levine G, Bates E, Blankenship J, et al. 2011 ACCF/AHA/SCAI Guideline for Percutaneous Coronary Intervention: A Repor of the the American College of Cardiology Foundation/American Heart Association Task Force on Practice Guidelines and the Society for Cardiovascular Angiography and Interventions. Circulation. 2011;124:e574-651. 
4. Park DW, Seung KB, Kim YH, et al. Long-term safety and efficacy of stenting versus coronary artery bypass grafting for unprotected left main coronary artery disease 5-year results from the MAIN-COMPARE (Revascularization for Unprotected Left Main Coronary Artery Stenosis: Comparison of Percutaneous Coronary Angioplasty Versus Surgical Revascularization) Registry. J Am Coll Cardiol. 2010;56:117-24.

5. Park SJ, Kim YH, Park DW, et al. Randomized trial of stents versus bypass surgery for left main coronary artery disease. $\mathrm{N}$ Engl J Med. 2011;364:1718-27.

6. Kim YH, Park DW, Ahn JM, et al. Everolimus-eluting stent implantation for unprotected left main coronary artery stenosis. The PRECOMBAT-2 (Premier of Randomized Comparison of Bypass Surgery versus Angioplasty Using Sirolimus-Eluting Stent in Patients with Left Main Coronary Artery Disease) Study. J Am Coll Cardiol Intv. 2012;5:708-17.

7. Mohr FW, Morice MC, Kappetein AP, et al. Coronary artery bypass graft surgery versus percutaneous coronary intervention in patients with three-vessel disease and left main coronary disease: 5-year follow-up of the randomizedMo, clinical SYNTAX trial. Lancet. 2013;381:629-38.

8. Montalescot G, Sechtem U, Achebach S, et al. 2013 ESC guidelines on the treatment of stable coronary disease. The task force on the management of stable coronary disease of the European Society of Cardiology. Eur Heart J. 2013;34:2949-3003.

9. Price MJ, Cristea E, Sawhney N, et al. Serial angiographic follow-up of sirolimus-eluting stents for unprotected left main coronary artery revascularization. J Am Coll Cardiol. 2006;47:871-7.

10. Park SJ, Kim YH, Park DW, et al. Impact of intravascular ultrasound guidance on long-term mortality in stenting for unprotected left main coronary artery stenosis. Circ Cardiovasc Interv. 2009;2:167-77.
11. Kim YH, Park SW, Hong MK, et al. Comparison of simple and complex stenting techniques in the treatment of unprotected left main coronary artery bifurcation stenosis. Am J Cardiol. 2006;97:1597-601.

12. Zingone B, Pappalardo A, Dreas L, et al. Logistic versus additive EuroSCORE. A comparative assessment of the two models in an independent population sample. Eur J Cardiothorac Surg. 2004;26:1134-40.

13. Sianos, Morel MA, Kappetein AP, et al. The SYNTAX Score: an angiographic tool grading the complexity of coronary artery disease. Eurolnterv. 2005;1:219-27.

14. Jou YL, Lu TM, Chen YH, et al. Comparison of the predictive value of EuroSCORE, SYNTAX score, and Clinical SYNTAX score for outcomes of patients undergoing percutaneous coronary intervention for unprotected left main coronary artery disease. Catheter Cardiovasc Inter. 2012;80:222-30.

15. Chang $\mathrm{K}$, Koh $\mathrm{Y}$, Jeong $\mathrm{S}$, et al. Long-term outcomes of percutaneous coronary intervention versus coronary artery bypass grafting for unprotected left main coronary bifurcation disease in the drug-eluting stent era. Heart. 2012;98:799e805.

16. Chieffo A, Meliga E, Latib A, et al. Drug-eluting stent for left main coronary artery disease the delta registry: a multicenter registry evaluating percutaneous coronary intervention versus coronary artery bypass grafting for left main treatment. J Am Coll Cardiol Intv. 2012;5:718-27.

17. Athappan G, Patvardhan E, Tuzcu M, et al. Left main coronary artery stenosis a meta-analysis of drug-eluting stents versus coronary artery bypass grafting. J Am Coll Cardiol Intv. 2013;6:1219-30.

18. Park DW, Kim YH, Yun SC, et al. Frequency, causes, predictors, and clinical significance of peri-procedural myocardial infarction following percutaneous coronary intervention. Eur Heart J. 2013;34:1662-9. 